\title{
Peripheral Nerve Growth within a Hydrogel Microchannel Scaffold Supported by a
}

\section{Kink-Resistant Conduit}

Dena Shahriari ${ }^{1}$, Masataka Shibayama ${ }^{2,3}$, Daniel Lynam ${ }^{4}$, Kayla Wolf ${ }^{4}$, Go Kubota ${ }^{2,3}$, Jacob Koffler ${ }^{5}$, Mark Tuszynski ${ }^{3,5,6}$, Wendy Campana ${ }^{2,3}$, Jeff Sakamoto ${ }^{1,7}$

${ }^{1}$ Department of Macromolecular Science and Engineering, University of Michigan, Ann Arbor, MI.

${ }^{2}$ Department of Anesthesiology \& ${ }^{3}$ Program in Neuroscience, University of California San Diego, La Jolla, CA.

${ }^{4}$ Department of Chemical Engineering and Materials Science, Michigan State University, East Lansing, MI

${ }^{5}$ Department of Neuroscience, University of California San Diego, La Jolla, CA.

${ }^{6}$ Veterans Administration Medical Center, La Jolla, CA.

${ }^{7}$ Department of Mechanical Engineering, University of Michigan, Ann Arbor, MI.

Keywords: Nerve repair; nerve regeneration; nerve guidance conduits; drilling hydrogels; agarose; poly caprolactone

\section{Significance}

1. Demonstrated a soft hydrogel can be micro-machined into scaffolds with high aspect ratio channels.

2. Developed a novel kink-resistant, suture-able nerve growth conduit capable of stabilizing and reinforcing the hydrogel microchannel scaffolds for peripheral nerve repair

3. Demonstrated the hybrid polymer conduit/hydrogel microchannel scaffold approach guides the linear penetration of axons and Schwann cells into $1 \mathrm{~cm}$ long rat sciatic nerve gaps.

\section{Abstract}

Nerve repair in several mm-long nerve gaps often requires an interventional technology.

Microchannel scaffolds have proven effective for bridging nerve gaps and guiding axons

in the peripheral nervous system (PNS). Nonetheless, fabricating microchannel scaffolds

at this length scale remains a challenge and/or is time consuming and cumbersome. In

this work, a simple computer-aided microdrilling technique was used to fabricate $10 \mathrm{~mm}$ -

long agarose scaffolds consisting of $300 \mu \mathrm{m}$-microchannels and $85 \mu \mathrm{m}$-thick walls in less

This is the author manuscript accepted for publication and has undergone full peer review but has not been through the copyediting, typesetting, pagination and proofreading process, which may lead to differences between this version and the Version record. Please cite this article as doi:10.1002/ jbm.a.36186. 
than an hour. The agarose scaffolds alone, however, did not exhibit adequate stiffness and integrity to withstand the mechanical stresses during implantation and suturing. To provide mechanical support and enable suturing, poly caprolactone (PCL) conduits were fabricated and agarose scaffolds were placed inside. A modified salt-leaching technique was developed to introduce interconnected porosity in PCL conduits to allow for tuning of the mechanical properties such as elastic modulus and strain to failure. It was shown that the PCL conduits were effective in stabilizing the agarose scaffolds in $10 \mathrm{~mm}$-long sciatic nerve gaps of rats for at least 8 weeks. Robust axon ingress and Schwann cell penetration were observed within the microchannel scaffolds without using growth factors.

\section{Introduction}

Peripheral nerve injury (PNI) occurs in 3\% of all trauma cases and $30 \%$ of combat injuries $^{(1)-(3)}$, which results in approximately 600,000 surgical procedures for PNI annually $^{(4)}$. Although spontaneous nerve regeneration can occur, functional restoration depends on the severity of the injury and is often limited. Interventional technology may be required to repair $\mathrm{cm}$-long injuries after acute nerve trauma. Autologous grafts are the current gold standard approach to treat PNI, but have practical limitations such as donor site morbidity and can result in painful neuromas ${ }^{(2),(5),(6)}$.

Bioengineered grafts are under development as an alternative therapy and several helpful review papers describe and compare different designs ${ }^{(7)-(11)}$. The goal of this approach is to circumvent issues related to donor availability while guiding axons in cmscale peripheral nerve gaps. Several types of bioengineered conduits such as 
Neurotube $^{\circledR(11)}$, Surgisis ${ }^{\circledR(12)-(14)}$, NeuroMatrix ${ }^{\circledR(11)}$, Neuroflex ${ }^{\circledR(15)}$ and NeuraGen ${ }^{\circledR(1),(3)}$ are currently approved by the USA FDA for PNI. There are, however, a few shortcomings that are yet to be addressed ${ }^{(11)}$. First, there are currently limitations in producing few $\mathrm{cm}-$ long conduits that correspond to clinical demands with the longest grafts being less than $6.5 \mathrm{~cm}$. Second, it is necessary to fabricate a flexible, kink-resistant conduit in order to prevent the compression of growing axons ${ }^{(11),(15)-(17)}$ that has been a challenge to achieve. For example, Neuroflex ${ }^{\circledR}$ is one of the most flexible FDA-approved conduits to-this-date, which is reported by the manufacturer as kink-free for up to $60^{\circ}$ of bending. However, Neuroflex ${ }^{\circledR}$ is not available in lengths more than $2.5 \mathrm{~cm}$. Overall, a more compliant nerve conduit is necessary to prevent or limit injuries caused by relatively stiff conduits. Lastly, the clinical efficacy of conduits is usually limited to nerve gaps less than $3 \mathrm{~cm}$. This could be because all synthetic FDA-approved PNI implants consist of single-lumen tubes. An alternative implant to more effectively guide axons is the use of multi-lumen microchannels that organize or group axons into fascicles ${ }^{(5),(7),(10),(11)}$ and guide them toward distal targets. Examples include multi-lumen microchannel scaffolds, which were demonstrated effective in linearly guiding axons in $2 \mathrm{~mm}$-long transected sections of the spinal cord ${ }^{(18)-(21)}$. Particularly, agarose microchannel scaffolds were produced using a fiber-optic templating technology and provided axon guidance in nerve gaps ${ }^{(18),(22),(23)}$. However, there are limitations in fabricating multiple cm-long templated agarose scaffolds ${ }^{(24)}$ and these processes may take up to two weeks to produce a scaffold and may require training to be reproduced by an individual. In addition, a PNI implant needs to be secured (for example be sutured to a nerve stump) in regions such as the sciatic nerve 
where muscle tissue undergoes significant displacement ${ }^{(11),(15)-(17)}$. To address these shortcomings, we aimed to produce a microchannel scaffold embedded inside a kinkresistant conduit that can be sutured to the nerve stumps in a rat sciatic nerve gap.

To fabricate few mm-long microchannel scaffolds, we used a simple subtractive mechanical technique (microdrilling) to produce agarose scaffolds with hexagonallyarranged cm-scale microchannels in less than an hour. The scaffolds consisted of $300 \mu \mathrm{m}$ diameter microchannels and $85 \mu \mathrm{m}$ walls. To our knowledge, this is the first report to demonstrate the successful drilling of micro-scale features in a hydrogel. To secure the microchannel scaffold to the nerve stumps, a suturable outer conduit was fabricated to fit around the agarose scaffold. This is because agarose hydrogel does not have adequate mechanical integrity to withstand suturing. The conduits consisted of poly caprolactone (PCL). PCL was selected as the conduit material since it is FDA-approved for applications such as sutures and it is known to be biocompatible with the nerve tissue $^{(25),(26)}$. However, dense PCL is brittle ${ }^{(27)}$ and not suitable for suturing. To enable suturing, porosity was introduced into the PCL using a simple salt-leaching technique. The porosity reduced the PCL elastic modulus (stiffness) and increased strain to failure, both of which make suturing facile. By introducing porosity into the PCL using a saltleaching technique, the strain to failure was increased from $17 \%$ to over $100 \%$. Moreover, the porous PCL conduit was kink-resistant when bent around fingertips; a property comparable to a flexible state-of-the-art PNI conduit- Neuroflex ${ }^{\circledR}$. In addition, a tube manufacturing process was developed to prepare relatively thin-wall PCL conduits 
(100 $\mu \mathrm{m})$ to minimize the conduit/scaffold construct volume. These tubes could be fabricated $>10 \mathrm{~cm}$ in length; longer than any FDA-approved PNI conduit.

When sutured to both sides of a $1 \mathrm{~cm}$-long transected rat sciatic nerve, the scaffold/conduit construct remained mechanically stable after 8 weeks in vivo and some axon and Schwann cell penetration into the proximal end of the scaffold was observed.

Overall, this work demonstrated the efficacy of a hybrid nerve guidance scaffold approach whereby a hydrogel microchannel scaffold (agarose) physically guided axons toward distal targets while a flexible outer conduit was sutured to nerve stumps and stabilized the scaffold in a highly mobile region of rat sciatic nerve for at least 8 weeks. It is suggested that the hybrid design is a promising alternative to autologous nerve grafts.

\section{Methods}

\subsection{Agarose scaffold fabrication}

Agarose hydrogel was prepared by heating agarose powder (3 wt.\%, Sigma Aldrich; St. Louis, MO) in autoclaved reverse osmosis (RO) water at $\sim 100^{\circ} \mathrm{C}$. To eliminate air bubbles, the agarose solution was centrifuged at 1500 rotations per minute (rpm) for $30 \mathrm{~s}$. The solution was poured into a sterile 12 well-plate (VWR; Radnor, PA) and cooled to room temperature in a sterile hood to form a hydrogel. After gelation, the cylindricallyshaped hydrogel cylinders were extracted from the well plate and machined to produce $10.0 \mathrm{~mm}$-tall cylinders. A computer numerical control (CNC) machine (US Digital, Vancouver, WA) was used to machine the height of the hydrogel cylinder using a $3.0 \mathrm{~mm}$ drill bit (McMaster, Aurora, IL) and create cylinders with parallel faces. 
To microdrill scaffolds, a $3 \times 8 \mathrm{~mm}$ piece of polystyrene (PS) (VWR) was attached to the top corner of a well of a 12-well plate (Fig. 1). To ensure the channels extended through the $10 \mathrm{~mm}$-tall cylinder, a sacrificial layer $(7 \mathrm{~mm}$-thick) of agarose hydrogel was prepared similar to the $10 \mathrm{~mm}$-tall cylinder and was placed at the bottom of the well plate. The $10 \mathrm{~mm}$-tall agarose cylinder was placed over the sacrificial agarose with the PS piece anchoring the cylinder and preventing it from spinning/moving during microdrilling. A $300 \mu \mathrm{m}$ (McMaster) microdrill bit was used to drill the agarose cylinder in a hexagonal arrangement with spacing of $100 \mu \mathrm{m}$ using the $\mathrm{CNC}$ and a patterning program (Artsoft Software Inc.; Livermore Falls, ME). A feed rate of $25.4 \mathrm{~mm} / \mathrm{min}$ was used to microdrill channels. After each $250 \mu \mathrm{m}$ of drilling, the bit was extracted from the agarose hydrogel disk to remove swarf (also known as peck drilling). Feed rates and peck drilling depths above and below the mentioned values did not result in linear channels or symmetric hexagonal patterning. A total depth of $11.6 \mathrm{~mm}$ was drilled (thus drilling into the sacrificial cylinder) to ensure the channels extended the entire length of the scaffold. A few $\mathrm{ml}$ of water was maintained above the hydrogel cylinder during drilling to prevent dehydration and help remove swarf from the channels and the microdrill bit. The agarose cylinder was drilled for at least $1.4 \times 1.4 \mathrm{~mm}$-wide surface to fabricate a scaffold with a diameter of $1.4 \mathrm{~mm}$. The entire process took less than 45 minutes to complete.

A customized sharpened punch with an inner diameter of $1.4 \mathrm{~mm}$ was used to extract agarose scaffold from the drilled cylinder. The punch was inserted perpendicular to the surface of the cylinder at a speed of $25.4 \mathrm{~mm} / \mathrm{min}$ for a depth of $14 \mathrm{~mm}$ using a 1 
mm diameter rod. Air was gently blown into the scaffold to remove swarf. The channel linearity and agarose scaffold dimensions were characterized using optical microscopy (Leica EZ4D; Buffalo Grove, IL). The scaffolds were sterilized and stored in RO water with 5 vol\% ethanol (Alfa Aesar; Ward Hill, MA) until implantation.

\subsection{PCL conduit fabrication and scaffold assembly}

Porosity was introduced into PCL using sodium chloride $(\mathrm{NaCl})$ particles as a porogen. $\mathrm{NaCl}$ (Columbus Chemical Industries, INC.; Columbus, WI) was ground using a planetary ball-mill (Retsch PM 100; Haan, Germany) in a $250 \mathrm{ml}$ agate jar with 12 agate balls for $1 \mathrm{hr}$ at $400 \mathrm{rpm}$ with $5 \mathrm{~min}$ rest every $5 \mathrm{~min}$. $\varepsilon$-caprolactone (MW: 114.14) (Sigma; St. Lois, MO) was dissolved in chloroform (Sigma) (3 wt.\%) and added to the ball-milled $\mathrm{NaCl}$ to produce a 30:70 vol\% of PCL: NaCl mixture. The mixture was ballmilled for $20 \mathrm{~min}$ at $400 \mathrm{rpm}$ with $5 \mathrm{~min}$ rest every $5 \mathrm{~min}$. The rest period prevented over-heating of the jar. To fabricate porous PCL conduits, a steel rod with an outer diameter of $1.4 \mathrm{~mm}$ was placed in the PCL/ $\mathrm{NaCl}$ mixture and air-dried. After the solvent (chloroform) evaporated ( $2 \mathrm{~min})$, the PCL-coated rod was immersed in methanol (Sigma) for $\sim 1 \mathrm{~min}$. The PCL tubes were removed from the rod and cut into $12 \mathrm{~mm}$-long tubes using a razor. The tubes were then placed in $\mathrm{RO}$ water with $5 \mathrm{vol} \%$ ethanol to remove $\mathrm{NaCl}$. PCL tubes used for implantation were further sterilized under $u v$ light for 15 min. The PCL conduit was bent around fingertips and optical images were taken (iPhone 5, Apple Inc.; Cupertino, CA).

Assembling the scaffolds entailed the insertion of the agarose scaffolds into the tubes. Agarose scaffolds (fabricated in section 2.1) were partially dehydrated with the 
touch of a kimwipe (Kimberly-Clack; Roswell, GA) to temporarily cause radial contraction and facilitate insertion into the tubes. Using a $1 \mathrm{~mm}$-diameter metal rod, dehydrated scaffolds were pushed into and manually centered in the $12 \mathrm{~mm}$-long PCL tubes with $1 \mathrm{~mm}$ overhangs at each end. Agarose scaffolds immediately returned to the original dimensions after rehydration in RO water. By dehydrating, inserting, and rehydrating, the outer diameter of the agarose scaffolds matched the inner diameter of the PCL tube, which significantly limited the scaffold movement inside the PCL tubes. The scaffolds were stored in 5 vol\% ethanol in RO water for up to 14 days and rinsed in sterile $1 \mathrm{xDPBS}$ buffer for 15 minutes prior to implantation.

\subsection{Characterizing the porosity and mechanical properties of PCL}

To characterize porosity, the salt-leached PCL tubes described in section 2.2. were fractured after immersion in liquid $\mathrm{N}_{2}$ followed by sputter coating with gold for $120 \mathrm{~s}$ (3 nm-thick coating) (SPI Supplies; West Chester, PA). The fracture surfaces were imaged using scanning electron microscopy (SEM) (FEI Nova NanoLab ${ }^{\mathrm{TM}} 600$ DualBeam; Columbus, $\mathrm{OH})$. In addition, a needle was passed through a salt-leached PCL tube in vitro to evaluate its suture-ability. The punctured PCL was gold-coated similar to the PCL tube and characterized with SEM.

Non-porous and porous PCL films were fabricated to characterize the mechanical properties of PCL. The 3 wt.\% PCL dissolved in chloroform and the PCL/NaCl mixture (described in section 2.2) was poured on copper foil (MTI Corporation; Richmond, CA) secured to an automated tape casting coater (MTI Corporation). A doctorblade (MTI Corporation) with a height of $50 \mu \mathrm{m}$ was used to evenly spread the solutions. The PCL 
films were air-dried. To delaminate the films from the copper foil, methanol (Sigma) was poured on top of the films. The film with $\mathrm{NaCl}$ was then placed in water for at least $12 \mathrm{hr}$ to remove $\mathrm{NaCl}$. PCL rectangular pieces $(25 \times 6 \mathrm{~mm})$ were cut using a razor, and tensile testing was performed with a $1.5 \mathrm{~N} / \mathrm{min}$ loading rate $(\mathrm{n}=5)($ Instron; Norwood, MA).

\subsection{In vivo testing}

Animals were housed 2-3 per cage with free access to food and water in a vivarium approved by the American Association for the Accreditation of Laboratory Animal Care. All animal studies were carried out according to protocols approved by the Institutional Animal Care and Use Committee of the VA Healthcare System, San Diego and following the IASP Guidelines for Use of Animals in Research. Adult male Sprague-Dawley rats (150-200 g) underwent surgery under anesthesia using ketamine $(25 \mathrm{mg} / \mathrm{ml})$, xylazine $(1300 \mathrm{mg} / \mathrm{ml})$ and acepromazine $(0.25 \mathrm{mg} / \mathrm{ml})$. The hindquarters were shaved and sterilized with ethanol (Sigma). Skin was incised parallel to the femur, and the sciatic nerve was exposed via a gluteal muscle-splitting approach. A 10-mm segment of sciatic nerve, $5 \mathrm{~mm}$ proximal to the sciatic trifurcation, was cut. A sterilized $10 \mathrm{~mm}$-long agarose scaffold inside a 12 mm-long PCL conduit (described in sections 2.1 and 2.3) (n =7) or a $12 \mathrm{~mm}$-long hollow PCL conduit $(\mathrm{n}=3)$ was inserted in each rat nerve gap and the PCL conduit was sutured to the ends of nerve stumps with two interrupted 9-0 nylon microsutures. Muscle was sutured with 5-0 polyglactin (Vicryl Ethicon Suture; Somerville, NJ), and the skin was stapled. Following surgery, rats received banamine (1 $\mathrm{mg} / \mathrm{kg})$ and ampicillin $(0.2 \mathrm{mg} / \mathrm{kg})$ in Ringer's lactate for three days. 
Paraformaldehyde (PFA) (4\%) was used to perfuse the animals eight weeks after implantation. The site of surgery was cut and the implant and the nerve were transferred to sucrose: $1 \mathrm{xDPBS}$ solution (30:70 wt. ratio) prior to dissection and sectioning. For evaluation of the implant site, $20 \mu \mathrm{m}$-thick longitudinal tissue sections were cryosectioned. The slides were treated with proteinase K:antigen retrieval $(1: 20)$ (Millipore; Darmstadt, Germany) for $20 \mathrm{~min}$. Subsequent washes were followed using $50 \%$ methanol for $10 \mathrm{~min}, 100 \%$ methanol for $20 \mathrm{~min}$ and $50 \%$ methanol for $5 \mathrm{~min}$. Later the slides were placed in 5\% of goat serum and Tris-buffered saline (TBS) for 20 min. Mouse-anti-Neurofilament 200 (1:500) (Millipore) and rabbit-anti-S100 protein (1:500) (Dako; Carpenteria, CA) were used to label neurofilaments and Schwann cells, respectively. Slides were washed with TBS and labeled with Goat-anti-mouse AlexaFluor 488 and Goat-anti-rabbit 594 (Life Technologies, Carlsbad, CA). Slides are visualized using an Olympus BX 53 microscope. Fluorescent images were taken with a mounted camera (Q imaging Retina 2000R) and CellSens Digital Imaging computer software.

\section{Results}

3.1. Agarose scaffold and PCL conduit characterization

The agarose scaffolds were $10.0 \mathrm{~mm}$ in length and $1.4 \mathrm{~mm}$ in outer diameter $(\mathrm{n}=5)$. Each microchannel diameter was $300 \pm 10 \mu \mathrm{m}$ with spacing of $85 \pm 5 \mu \mathrm{m}$ (average \pm SD) (Fig. 2(a)). The spacing between the channels (specified as $100 \mu \mathrm{m}$ on the CNC) was the smallest spacing that resulted in continuous linear channels when microdrilling the agarose and was therefore used to maximize the lumen volume of the scaffold. Attempts 
in reducing the wall thickness to increase the lumen volume resulted in scaffolds with inadequate mechanical integrity to withstand implantation. To ensure microchannel linearity, longitudinal images were analyzed (Fig. 2(b)). The linear features in Fig. 2(b) represent the microchannels, which appear continuous and regular along the entire scaffold length. The optical analysis confirms that the microdrilling process was successful in fabricating microchannel agarose scaffolds with relatively thin walls $(85 \pm 5$ $\mu \mathrm{m})$ and high aspect ratio channels (33:1), but using a mechanical process rather than previously introduced chemical removal process ${ }^{(18)}$. Unlike for example fiber templating processes, microdrilling one scaffold was completed in less than an hour and could be performed by an individual without a special training.

The agarose microchannel scaffolds did not have sufficient mechanical properties to allow for their suturing to nerve stumps. Thus, PCL conduits were developed to mechanically reinforce the agarose scaffolds (Fig. 2(c)). To better understand the mechanical behavior, PCL mechanical property characterization was conducted and separated into two sections described below: stress-strain behavior and flexibility.

\subsubsection{Characterizing conduit porosity and mechanical properties}

To assess the mechanical integrity of PCL against rupturing and to characterize the effect of porosity on this property, tensile testing and SEM was performed. The elastic moduli of non-porous PCL and 70 vol\% porous PCL were $182.15 \pm 17.46 \mathrm{MPa}$ and $2.09 \pm 0.34$ $\mathrm{MPa}$ (average $\pm \mathrm{SD}, \mathrm{n}=5$ ), respectively. In addition, using tensile testing, the strain to failure of PCL with and without porosity was obtained (Fig. 2(d) and Fig. 2(e)). Strain to failure is the strain\% (percentage of change in length) before rupture and was selected as 
the characterization method to assess the performance of PCL under tension in vivo. Non-porous PCL ruptured at $17.9 \pm 1.3 \%$ strain while 70 vol\% porous PCL (crosssectional SEM image shown in Fig. 2(f)) ruptured at $101.8 \pm 12.2 \%$ strain (average \pm SD, $\mathrm{n}=5$ ). In addition, while non-porous PCL tore upon puncturing with a suturing needle, porous PCL with a thickness comparable to non-porous PCL did not tear and its morphology surrounding the punctured site was not disturbed (Fig. 2(g)). The increase in the strain to failure of PCL with increased porosity may be the reason for the improved suture-ability of the material when porosity is introduced. Overall, it was determined that porous PCL had adequate mechanical integrity to allow suturing. Therefore, only porous conduits were selected for the rest of the study.

\subsubsection{PCL conduit flexibility}

The PCL conduits were fabricated in lengths $>10 \mathrm{~cm}$ and exhibited flexibility comparable to state-of-the-art nerve guidance conduits ${ }^{(11)}$. PCL conduit flexibility was demonstrated by measuring the maximum bend angle before a kink occurred. As demonstrated in Fig. 2(h), the tubes were kink-free up to a bend angle of $58^{\circ}$ exhibiting a bend radius of $\sim 7.5 \mathrm{~mm}$. Based on their flexibility and kink-resistance property, the PCL conduits were comparable to state-of-the-art, FDA-approved nerve guidance conduits.

\subsection{Scaffold nerve stump integration}

Microchannel agarose scaffolds placed inside PCL conduits were implanted in $10 \mathrm{~mm}$ long rats sciatic nerve gaps (Fig. 3(a)). The agarose scaffolds were $10 \mathrm{~mm}$ long and were inserted into $12 \mathrm{~mm}$ long PCL conduits. The additional PCL conduit length created 1 mm overhangs (cuff) on each end of the implant. The overhangs enabled suturing of the 
scaffolds to the ends of the nerve stump. After 8 weeks of implantation, the scaffold remained integrated with the nerve, demonstrating the efficacy of the PCL tube in stabilizing a scaffold in the highly mobile region of sciatic nerve (Fig. 3(b) and 3(c)). In addition, the PCL tubes and agarose scaffolds maintained their mechanical integrity and no signs of degradation were visible. The overhangs allowed for suturing of the nerve stumps to the scaffold. Such design has been previously used by for example Huang et al. when PGA Neurotube ${ }^{\mathrm{TM}}$ conduits with $3 \mathrm{~mm}$-long overhangs were sutured to the nerve stumps of $10 \mathrm{~mm}$-long rat sciatic nerve gaps using 4 sutures on each side ${ }^{(28)}$. The conduits remained stable for at least 8 weeks confirming the advantages of overhangs in stabilizing scaffolds to nerve stumps.

\subsection{In vivo characterization}

Animals were perfused eight weeks post-implantation and longitudinal slides of the scaffolds were obtained. The longitudinal view of the proximal end showed axons and Schwann cells were present in about $25 \%$ of the scaffold channels of all animals $(n=7)$ (Fig. 3(d-f)). Neural growth was along the longitudinal axis of the channels. In addition, axons and Schwann cells grew within the $10 \mathrm{~mm}$-long scaffold channels and egressed from the scaffolds into the host tissue for three out of the seven animals (Fig. 3(g-i)). To validate the efficacy of microchannel scaffolds for bridging nerve gaps and to confirm that the presence of axons and Schwann cells at the distal ends of the scaffolds is primarily due to the presence of linear channels, axon growth within hollow PCL conduits implanted in $10 \mathrm{~mm}$-long sciatic nerve gaps was assessed. Fig. 4(a) is a representative image of axons at the proximal end of a PCL conduit that did not contain 
an agarose microchannel scaffold. Axon penetration into the conduit at the proximal end was observed, but axon growth was not oriented and axons were scarcely present at the distal end (Fig. 4(b)).

\section{Discussion}

A computer-controlled microdrill was used to fabricate agarose hydrogel scaffolds consisting of $1 \mathrm{~cm}$-long microchannels. To provide mechanical support, a robust PCL outer conduit technology was introduced to create kink-resistant conduits. The conduit reinforced the agarose microchannel scaffolds and enabled suturing of the PCL conduits to peripheral nerve stumps. While few cm-long PCL conduits could be easily fabricated, it is recognized that microdrilling agarose scaffolds longer than $1.5 \mathrm{~cm}$ may be challenging. Thus, for nerve gaps larger than $1.5 \mathrm{~cm}$, one possibility is to stack multiple agarose scaffolds inside a continuous PCL conduit to provide few cm-long scaffold/conduit construct. Overall, this investigation demonstrated that the combination of synthetic microchannel scaffolds and outer protective conduits could be a viable alternative to autologous nerve implants.

In vivo testing in the sciatic nerve model demonstrated that the agarose microchannel scaffold/PCL nerve guidance implants were effective in linearly guiding and organizing axons and Schwann cells. When implanted in rat sciatic nerves, linear neural cell growth through the length of some of the scaffold channels confirmed the microchannel construct provided a structure that, compared to hollow conduits, was more effective in linearly guiding axons through the nerve gap and maintaining their organization. While not all the scaffolds provided full growth of axons and Schwann 
cells through the entire $1 \mathrm{~cm}$ nerve gap, we believe that the axon and Schwann cell density and degree of axon growth can be significantly augmented if growth factors for axon and Schwann cell growth were present. Providing a longer regeneration time (more than the experimented 8 weeks) may also improve neural growth to the distal end. Future work will involve filling scaffolds with growth factors and increasing the experimental time in efforts to significantly improve axon growth and recapitulation.

\section{Acknowledgement}

The funding agencies, the USA NIH (R01 EB014986), the Veterans Administration, Dr. Miriam and Sheldon G. Adelson Medical Research Foundation as well as the National Science Foundation for a Graduate Student Fellowship to DS are greatly appreciated.

\section{Disclosure}

In a separate study, the PCL tube technology was modified and advanced to all-PCL microchannel scaffolds. This technology may be licensed to a product called ReAxon cofunded by JSS, MTH, WMC and JYK.

\section{References}

1. Archibald SJ, Shefner J, Krarup C, Madison RD. Monkey median nerve repaired by nerve graft or collagen nerve guide tube. The Journal of Neuroscience. 1995;15:4109-23.

2. Beltran MJ, Burns TC, Eckel TT, Potter BK, Wenke JC, Hsu JR. Fate of Combat Nerve Injury. Journal of Orthopaedic Trauma. 2012;26:e198-e203.

3. Krarup C, Archibald SJ, Madison RD. Factors that influence peripheral nerve regeneration: An electrophysiological study of the monkey median nerve. Annals of Neurology. 2002;51:69-81.

4. Brattain K. Analysis of the peripheral nerve repair market in the United States. contentstockprcom. 2012.

5. Ueyama T. The topography of root fibres within the sciatic nerve trunk of the dog. 
Journal of Anatomy. Wiley-Blackwell; 1978;127:277.

6. $\quad$ Li R, Liu Z, Pan Y, Chen L, Zhang Z, Lu L. Peripheral Nerve Injuries Treatment: a Systematic Review. Cell Biochem Biophys. Springer US; 2013;68:449-54.

7. Heath CA, Rutkowski GE. The development of bioartificial nerve grafts for peripheral-nerve regeneration. Trends in Biotechnology. 1998;16:163-8.

8. Belkas JS, Shoichett MS, Midha R. Peripheral nerve regeneration through guidance tubes. Neurological Research. 2004;26:151-60.

9. Ichihara S, Inada Y, Nakamura T. Artificial nerve tubes and their application for repair of peripheral nerve injury: an update of current concepts. Injury. 2008;39:29-39.

10. Gu X, Ding F, Yang Y, Liu J. Construction of tissue engineered nerve grafts and their application in peripheral nerve regeneration. Progress in Neurobiology. 2011;93:204-30.

11. Kehoe S, Zhang XF, Boyd D. FDA approved guidance conduits and wraps for peripheral nerve injury: A review of materials and efficacy. Injury. 2012;43:55372.

12. Hadlock TA, Sundback CA, Hunter DA, Vacanti JP, Cheney ML. A new artificial nerve graft containing rolled Schwann cell monolayers. Microsurgery. 2001;21:96-101.

13. Smith RM, Wiedl C, Chubb P, Greene CH. Role of Small Intestine Submucosa (SIS) as a Nerve Conduit: Preliminary Report. J Invest Surg. 2004;17:339-44.

14. Hiles M, Hodde J. Tissue engineering a clinically useful extracellular matrix biomaterial. Int Urogynecol J. 2006;17:39-43.

15. Li S-T, Yuen D. Implant devices for nerve repair. US Patent. US Patent Office; 2004.

16. Meek MF, Coert JH. US Food and Drug Administration/Conformit EuropeApproved Absorbable Nerve Conduits for Clinical Repair of Peripheral and Cranial Nerves. Annals of Plastic Surgery. 2008;60:110-6.

17. de Ruiter GCW, Malessy MJA, Yaszemski MJ, Windebank AJ, Spinner RJ. Designing ideal conduits for peripheral nerve repair. Neurosurgical Focus. NIH Public Access; 2009;26:E5.

18. Stokols S, Sakamoto J, Breckon C, Holt T, Weiss J, Tuszynski MH. Templated Agarose Scaffolds Support Linear Axonal Regeneration. Tissue Engineering: Part 
A. $2006 ; 12: 2777-87$.

19. Pawar K, Mueller R, Caioni M, Prang P, Bogdahn U, Kunz W, Weidner N. Increasing capillary diameter and the incorporation of gelatin enhance axon outgrowth in alginate-based anisotropic hydrogels. Acta Biomaterialia.

2011;7:2826-34.

20. Thomas AM, Kubilius MB, Holland SJ, Seidlits SK, Boehler RM, Anderson AJ, Cummings BJ, Shea LD. Channel density and porosity of degradable bridging scaffolds on axon growth after spinal injury. Biomaterials. 2013;34:2213-20.

21. Shahriari D, Koffler J, Lynam DA, Tuszynski MH, Sakamoto JS. Characterizing the degradation of alginate hydrogel for use in multilumen scaffolds for spinal cord repair. J Biomed Mater Res. 2015;104:611-9.

22. Gros T, Sakamoto JS, Blesch A, Havton LA, Tuszynski MH. Regeneration of long-tract axons through sites of spinal cord injury using templated agarose scaffolds. Biomaterials. 2010;31:6719-29.

23. Gao M, Lu P, Bednark B, Lynam D, Conner JM, Sakamoto J, Tuszynski MH. Templated agarose scaffolds for the support of motor axon regeneration into sites of complete spinal cord transection. Biomaterials. 2013;34:1529-36.

24. Lynam D, Bednark B, Peterson C, Welker D, Gao M, Sakamoto JS. Precision microchannel scaffolds for central and peripheral nervous system repair. J Mater Sci: Mater Med. 2011;22:1-12.

25. Dunnen den WFA, Robinson PH, van Wessel R, Pennings AJ, van Leeuwen MBM, Schakenraad JM. Long-term evaluation of degradation and foreign-body reaction of subcutaneously implanted poly(DL-lactide-e-caprolactone). J Biomed Mater Res. 1997;36:337-46.

26. Chiang H-Y, Chien H-F, Shen H-H, Yang J-D, Chen Y-H, Chen J-H, Hsieh S-T. Reinnervation of Muscular Targets by Nerve Regeneration through Guidance Conduits. Journal of Neuropathology \& Experimental Neurology. 2005;64:576.

27. German RM. Powder Metallurgy Science. Metal Powder Industry; 1994. 1 p.

28. Huang JH, Cullen DK, Browne KD, Groff R, Zhang J, Pfister BJ, Zager EL, Smith DH. Long-Term Survival and Integration of Transplanted Engineered Nervous Tissue Constructs Promotes Peripheral Nerve Regeneration. Tissue Engineering Part A. 2009; 15:1677-85.

\section{Figure Captions:}


Fig. 1. Steps in microdrilling agarose scaffolds. (a) A sacrificial agarose disk is placed at the bottom of the well of a well-plate. An agarose disk with a height equivalent to the desired height of the scaffold (10 $\mathrm{mm}$ in the present study) was placed over the sacrificial layer. (b) A CNC-controlled microdrilling linear channels in the agarose disk. (c) A titanium tube was sharpened to a knife-edge and was used to cut scaffolds. (d) The scaffolds with linear channels were extracted from the sharpened punch.

Fig. 2. Agarose microchannel scaffold and PCL conduit characterization. (a) Crosssection of the agarose scaffold shows an array of $300 \mu \mathrm{m}$ inner diameter channels and 85 $\mu \mathrm{m}$ thick walls. The solid arrow points to the PCL conduit around the agarose scaffold and the dashed arrow points the interstitial channels created by the conduit around the scaffold. (b) The longitudinal view of an agarose scaffold consisted of $10 \mathrm{~mm}$-long linear channels. (c) The longitudinal view of the PCL conduit. (d) The stress-strain behavior of non-porous and porous PCL. The dashed line is the stress-strain curve for $100 \%$ non-porous PCL and the solid line is the curve for 70 vol\% porosity PCL. (e) The stress-strain behavior of 70 vol\% porosity PCL. (f) The SEM image of the PCL fracture surface. (g) The SEM image of a PCL conduit after a simulated in vitro suturing. (h) PCL tube bent around fingertips. The scale bars are as following: (a) is $0.3 \mathrm{~mm}$, (b) and (c) are $10 \mathrm{~mm},(\mathbf{f})$ is $0.02 \mathrm{~mm},(\mathbf{g})$ is $1 \mathrm{~mm}$ and (h) is $10 \mathrm{~mm}$.

Fig. 3. In vivo testing and efficacy of conduit/scaffold construct. (a) Orientation and location of the implantation. (b, c) The scaffold/conduit 8 weeks post-implantation was integrated with host tissue and remained intact: (b) in the nerve tissue and (c) dissected 
from the nerve tissue. The conduit was sutured to the host nerve using two sutures at each end as shown with solid arrows. Some capillary formation was observed at the distal end as indicated by the dashed arrow. The penetration of axons and Schwann cells into the proximal site of a scaffold channel in shown in figures (d) through (f) using NF200 (red) for axons and S100 (green) with (d) as the superimposed image, (e) showing axon penetration and (f) showing Schwann cell presence. The arrows demonstrate the direction of axon and Schwann cell penetration. Images of the proximal site are representative of all the seven tested animals. Figures (g) through (i) show the distal end of the scaffold with (g) axons and Schwann cells superimposed, (h) axons and (i) Schwann cells. The arrows demonstrate the direction of cell egress. Images of the distal end are representative of three out of the seven tested animals. The scale bars in (b) and (c) are $10 \mathrm{~mm}$. All other scale bars are $0.1 \mathrm{~mm}$.

Fig. 4. In vivo performance of a hollow PCL tube. Axon immunoreactivity with NF200 (red) in an implanted hollow 12 mm-long PCL conduit demonstrate (a) the penetration of axons into the scaffold at the proximal site and their growth at random orientations. (b) Relative to the proximal end, limited number of axons is present at the distal end of the conduit. Images are representative of all the three animals. The scale bars are $0.5 \mathrm{~mm}$. 


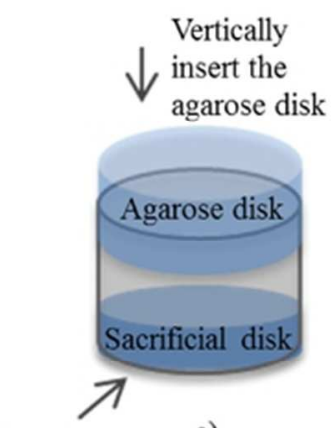

Well-plate

a)

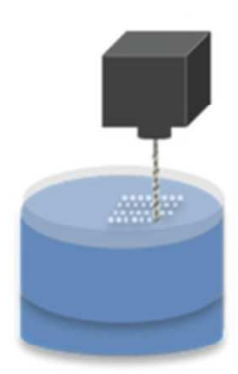

b)

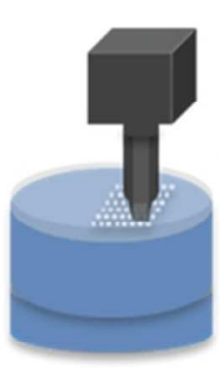

c)

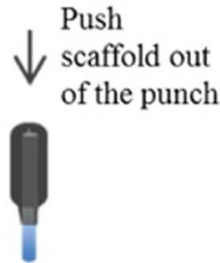

d)

Fig. 1. Steps in microdrilling agarose scaffolds. (a) A sacrificial agarose disk is placed at the bottom of the well of a well-plate. An agarose disk with a height equivalent to the desired height of the scaffold $(10 \mathrm{~mm}$ in the present study) was placed over the sacrificial layer. (b) A CNC-controlled microdrilling linear channels in the agarose disk. (c) A titanium tube was sharpened to a knife-edge and was used to cut scaffolds. (d) The scaffolds with linear channels were extracted from the sharpened punch.

$59 \times 22 \mathrm{~mm}(300 \times 300 \mathrm{DPI})$

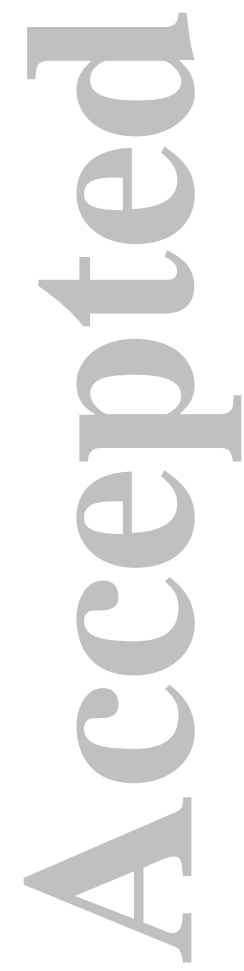

John Wiley \& Sons, Inc.

This article is protected by copyright. All rights reserved. 

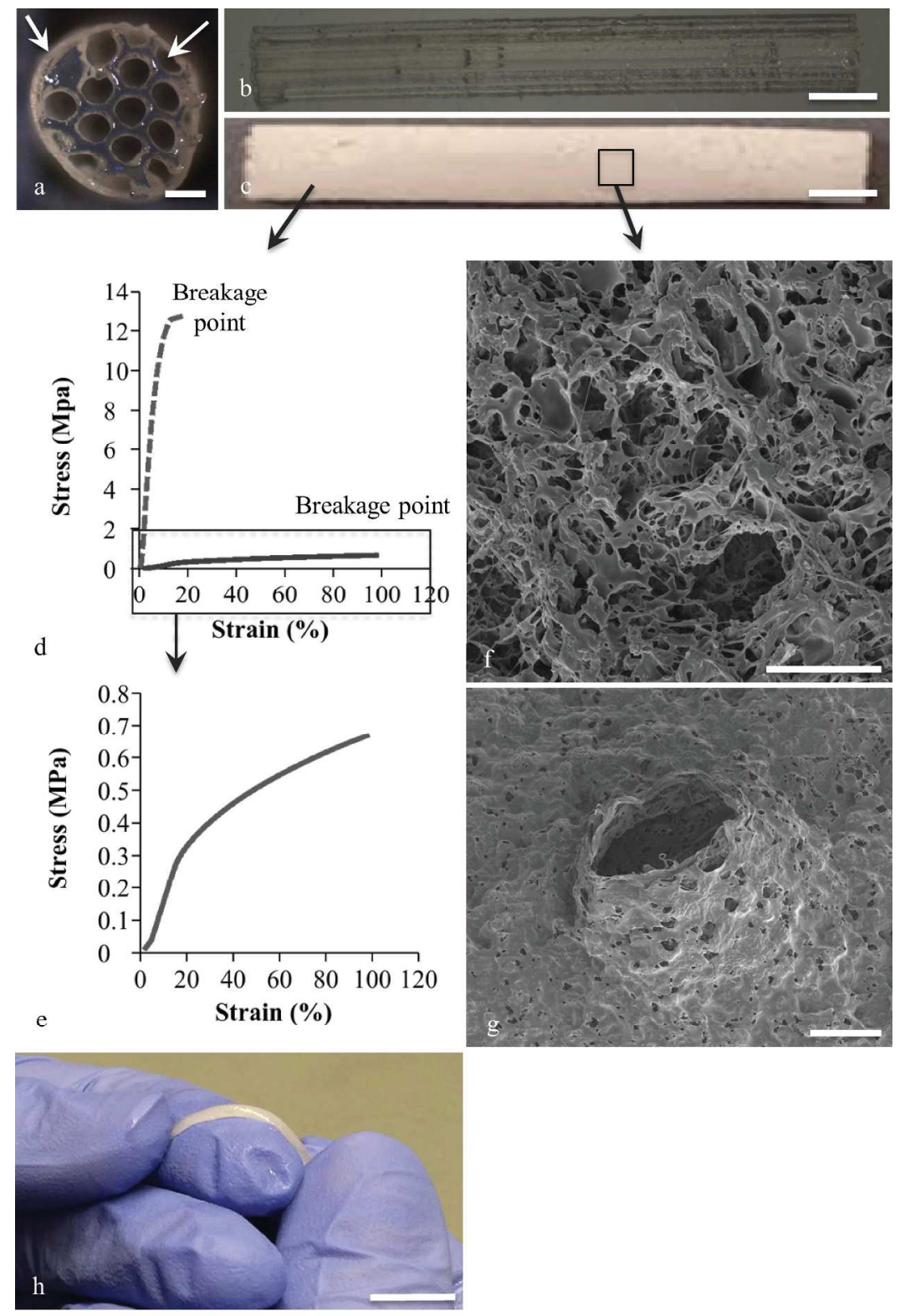

Fig. 2. Agarose microchannel scaffold and PCL conduit characterization. (a) Cross-section of the agarose scaffold shows an array of $300 \mu \mathrm{m}$ inner diameter channels and $85 \mu \mathrm{m}$ thick walls. The solid arrow points to the PCL conduit around the agarose scaffold and the dashed arrow points the interstitial channels created by the conduit around the scaffold. (b) The longitudinal view of an agarose scaffold consisted of $10 \mathrm{~mm}$-long linear channels. (c) The longitudinal view of the PCL conduit. (d) The stress-strain behavior of non-porous and porous PCL. The dashed line is the stress-strain curve for $100 \%$ non-porous PCL and the solid line is the curve for 70 vol\% porosity PCL. (e) The stress-strain behavior of 70 vol\% porosity PCL. (f) The SEM image of the PCL fracture surface. $(\mathrm{g})$ The SEM image of a PCL conduit after a simulated in vitro suturing. (h) PCL tube bent around fingertips. The scale bars are as following: (a) is $0.3 \mathrm{~mm}$, (b) and (c) are $10 \mathrm{~mm}$, (f) is $0.02 \mathrm{~mm},(\mathrm{~g})$ is $1 \mathrm{~mm}$ and $(\mathrm{h})$ is $10 \mathrm{~mm}$.

$198 \times 295 \mathrm{~mm}(300 \times 300 \mathrm{DPI})$

John Wiley \& Sons, Inc. 

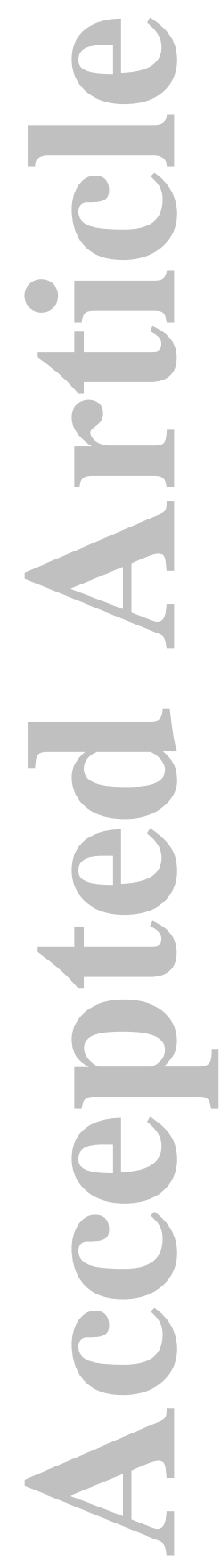

John Wiley \& Sons, Inc.

This article is protected by copyright. All rights reserved. 


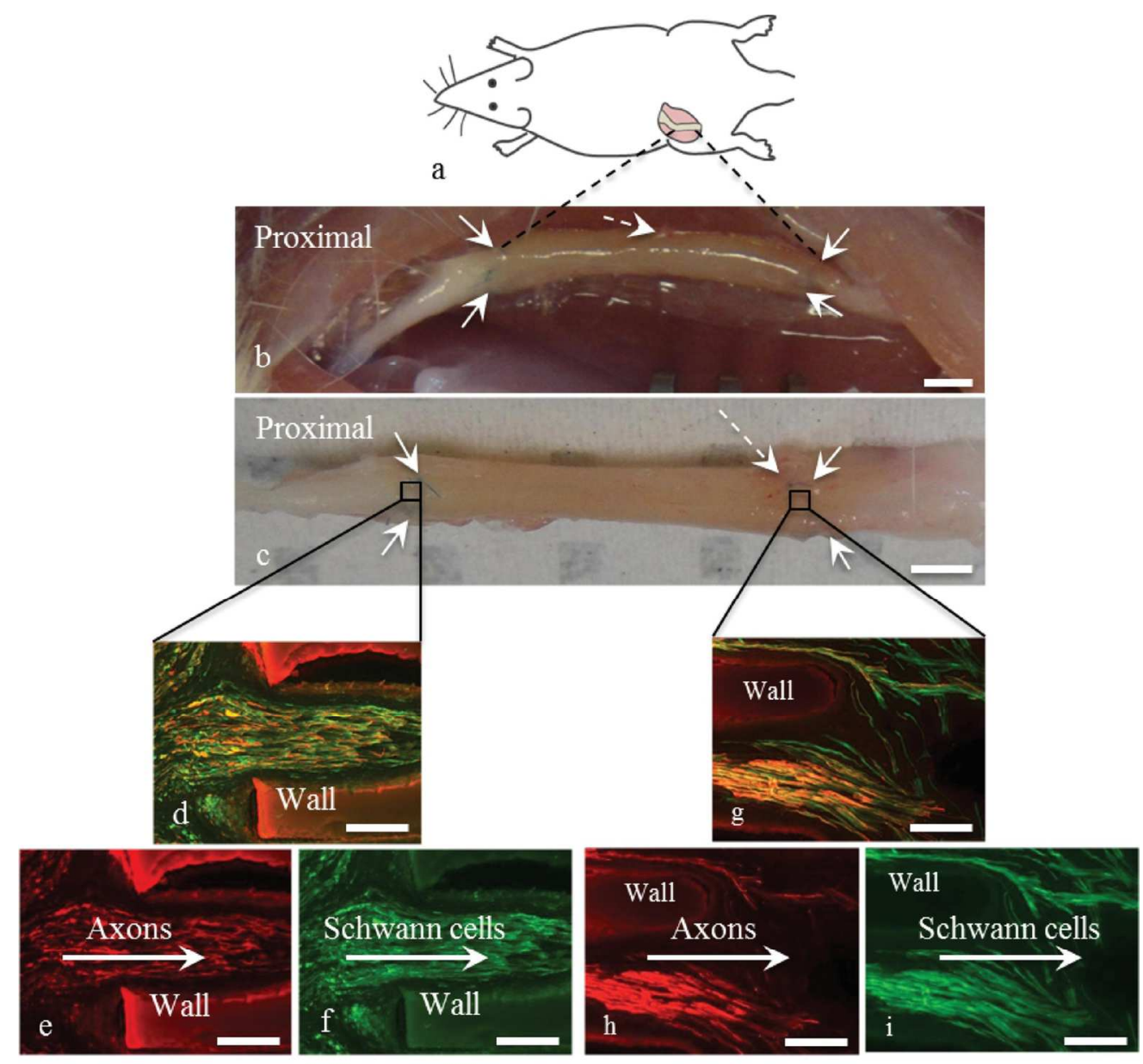

Fig. 3. In vivo testing and efficacy of conduit/scaffold construct. (a) Orientation and location of the implantation. (b, c) The scaffold/conduit 8 weeks post-implantation was integrated with host tissue and remained intact: (b) in the nerve tissue and (c) dissected from the nerve tissue. The conduit was sutured to the host nerve using two sutures at each end as shown with solid arrows. Some capillary formation was observed at the distal end as indicated by the dashed arrow. The penetration of axons and Schwann cells into the proximal site of a scaffold channel in shown in figures (d) through (f) using NF200 (red) for axons and S100 (green) with (d) as the superimposed image, (e) showing axon penetration and (f) showing Schwann cell presence. The arrows demonstrate the direction of axon and Schwann cell penetration. Images of the proximal site are representative of all the seven tested animals. Figures $(\mathrm{g})$ through (i) show the distal end of the scaffold with ( $g$ ) axons and Schwann cells superimposed, (h) axons and (i) Schwann cells. The arrows demonstrate the direction of cell egress. Images of the distal end are representative of three out of the seven tested animals. The scale bars in (b) and (c) are $10 \mathrm{~mm}$. All other scale bars are $0.1 \mathrm{~mm}$.

$$
126 \times 118 \mathrm{~mm}(300 \times 300 \text { DPI) }
$$




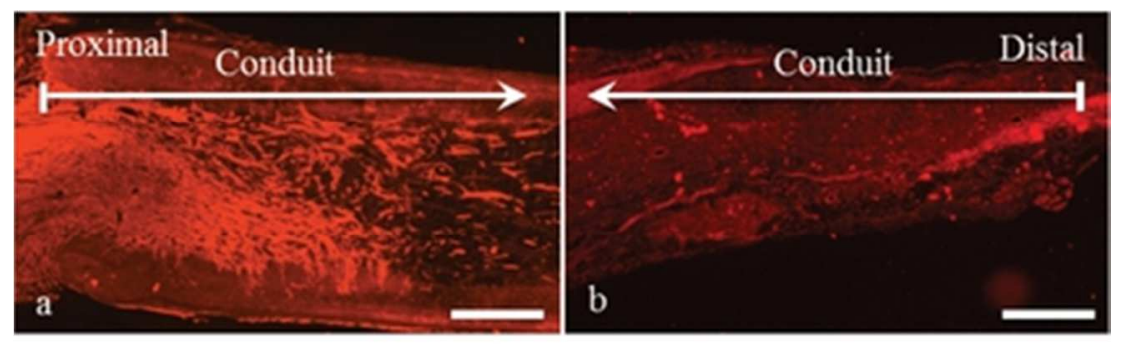

Fig. 4. In vivo performance of a hollow PCL tube. Axon immunoreactivity with NF200 (red) in an implanted hollow $12 \mathrm{~mm}$-long PCL conduit demonstrate (a) the penetration of axons into the scaffold at the proximal site and their growth at random orientations. (b) Relative to the proximal end, limited number of axons is present at the distal end of the conduit. Images are representative of all the three animals. The scale bars are $0.5 \mathrm{~mm}$.

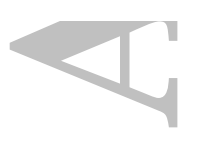

$35 \times 10 \mathrm{~mm}(300 \times 300$ DPI $)$

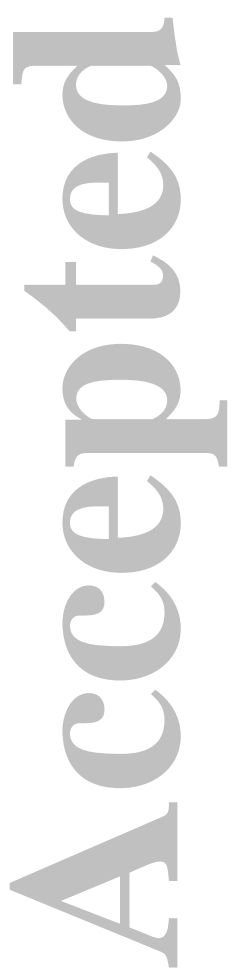

John Wiley \& Sons, Inc.

This article is protected by copyright. All rights reserved. 